\title{
Confocal imaging, visualization and 3-D surface measurement of small mammalian teeth
}

\author{
A. R. EVANS*, I. S. HARPER*† \& G. D. SANSON* \\ *Department of Biological Sciences and †Microscopy and Imaging Research Facility, Faculty of \\ Medicine, Monash University 3800, Australia
}

Key words. Confocal microscopy, GIS, measurement accuracy, morphometrics, moulding and casting, surface noise measurement, VRML animation.

\section{Summary}

The difficulties traditionally faced by functional morphologists in representing and interpreting three-dimensional objects can now be mostly overcome using available laser and computer imaging technologies. A practical method for three-dimensional imaging of small mammalian teeth using confocal microscopy is reported. Moulding and casting of the teeth were first performed, followed by confocal fluorescence imaging. Accuracy and precision of the scanned structures were tested in morphometric studies by using a new technique to measure the noise in the scan of a three-dimensional surface, and linear and angular dimensions of the scans were compared with measurements made using traditional morphological tools. It is shown that measurements can be taken with less than $4 \%$ difference from the original object. Teeth of the microchiropteran bat Chalinolobus gouldii were scanned and measured to show the potential of the techniques. Methods for visualizing the small teeth in three-dimensional space, and animating the teeth in occlusion, show the power of this approach in aiding a three-dimensional understanding of the structure and function of teeth and other three-dimensional structures.

\section{Introduction}

The functional morphologist has often been limited to representing anatomy as a two-dimensional projection - a photograph or drawing on paper. Proportions and spatial arrangements of components can easily be distorted or obscured in the abstraction from three to two dimensions, and so interpretations of projected images obviously should be, and usually are, undertaken with care. Representation and measurement of the object in its original three

Correspondence: Alistair Evans. Tel.: + 6139905 5655; fax: + 6139905 5613; e-mail: alistair.evans@sci.monash.edu.au dimensions is preferable. Therefore, an alternative approach is to digitize the object (represent the surface of the object by many discrete points in space) and manipulate the threedimensional surface data in virtual space with appropriate software. The ability to carry out the preferred alternative, particularly for very small specimens that cannot easily be inspected and manipulated without aid, has become a possibility with the advent of laser and computer technology in the form of the laser scanning confocal microscope.

Three-dimensional morphological analysis of teeth is beginning to gain popularity as the technology becomes more available (Reed, 1997; Zuccotti et al., 1998; Ungar \& Williamson, 2000), and the use of the confocal microscope for this work has begun on both microrelief (Boyde \& Fortelius, 1991; Boyde \& Jones, 1995) and macrorelief (Hunter \& Jernvall, 1998; Evans et al., 1999; Jernvall \& Selänne, 1999; Evans \& Sanson, 2000) of tooth surfaces. The technology of confocal microscopy, where a laser point source and confocal optics are used to sample an object point by point in 3-D space, is generally directed towards optical sectioning and 3-D reconstruction of tissues, cells and organelles, but other novel applications continue to emerge. Thus, we and colleagues have found it possible rapidly and accurately to map the surface (topology) of small teeth in a fraction of the time previously taken using manual measurements with, for example, a Reflex microscope. However, there are some important issues that must be addressed to ensure accurate and consistent results using these methods. This paper describes techniques that give practical solutions to these problems.

The technique described here involves incorporating a fluorescent dye, namely eosin, into the cast of a tooth. Fluorescence confocal imaging is then used to gather the three-dimensional surface information of the tooth. Once information representing the surface of an object such as a tooth has been gathered, a greater range of measurements can be taken compared to the usual point-to-point 
measures. Three-dimensional tooth shape can be measured, as well as areas and volumes, and the spatial relations between objects (i.e. how three-dimensional objects fit together) can be demonstrated. This means that the occlusion of the teeth can be visualized, which would normally be particularly difficult for small specimens when simply manipulating them by hand. Features highly relevant to tooth function can be identified and quantified, not just those features that are easy or convenient to measure, permitting the interpretation of the role of both the gross and finer-scale features in tooth function.

Confocal microscopy allows the reconstruction of surface topology from optical sections either by reflection or by fluorescence imaging. Reflection imaging of the teeth themselves, or gold sputter-coated replicas, would be ideal, but it was found that reflection imaging had a number of disadvantages. For original specimens, excessive reflection was observed at many positions over the surface, most likely due to enamel prisms or small sections of tooth surface perpendicular to the laser source causing significantly more reflection than the rest of the surface. This led to the misinterpretation of the surface position at these points of high reflection. Also, much less reflected light is obtained from a surface nearly parallel to the light direction (Cheng \& Kriete, 1995), again making the interpretation of surface position inconsistent for the whole tooth. The advantage of fluorescence in this respect is that fluorescence photons can go in any direction, increasing the likelihood that light will be received by the objective. Internal reflection within the microscope is also often a problem: most commercial confocal microscopes for biology are not equipped with anti-reflection coatings or internal anti-reflection optics, and this renders them inadequate for imaging in reflection mode. Staining the surface of a cast with fluorescent eosin gave the strongest signal compared to the low amount of autofluorescence exhibited by real teeth and was more accurate than depositing a layer of eosin dissolved in oil on the surface.

While measurements on a digital model can be made with a high degree of precision (e.g. Valeri et al., 1998), it is necessary to show that the digital model accurately represents the original. In the processes of moulding and casting and in imaging, a number of sources of error are introduced. These include shrinkage and distortion in moulding and casting, and depiction of the surface in imaging. In order to test adequately the confocal method, a standard test specimen of glass with known linear dimensions and angles was constructed. It was then moulded and cast, and the casts measured independently with a Reflex microscope or profile projector to show how faithful the cast was to the original. The cast was then imaged, digitally reconstructed and measured.

The design of the glass specimen also allowed the testing of different methods of imaging to determine which had the least error or noise in the scan of a surface. In this paper, 'surface noise' shall be defined as the deviation of the digital surface from the true surface at each $x, y$ point. As the top surface of the test specimen was a perfect plane, any deviations from the plane in the digital model represented surface noise and could easily be quantified.

To demonstrate the applicability of these techniques in a biological context, scans were made of teeth from three specimens of a bat, Chalinolobus gouldii, the molars of which are in the order of $2 \times 2 \mathrm{~mm}$. Morphological measurements taken from the specimens using digital calipers, a common tool in morphometric studies, showed that the lengths of individual molars were very similar, and so in most studies would often be considered identical in terms of function. However, minute differences in tooth shape between individuals can be demonstrated and measured using confocal and computer technology, meaning that fine differences in function can now be discerned and quantified.

\section{Materials and methods}

\section{Moulding}

All specimens were cleaned with a small brush using 100\% acetone, $100 \%$ ethanol and distilled water in succession, and allowed to air dry between each cleaning. Moulds were made of the upper half of a small glass specimen (about $1.7 \times 1.7 \mathrm{~mm}$ and $3 \mathrm{~mm}$ in height; Fig. 1) using silicone RTV 3110 (Dow Corning Corporation, Midland, U.S.A.). The glass specimen was first coated in a dilute soap solution, which then was allowed to dry, to prevent adhesion between the very smooth surface of the glass and the silicone.

The entire tooth rows of three specimens of Gould's wattled bat Chalinolobus gouldii (Vespertilionidae) (Museum Victoria specimens C3746 to C3748) were moulded and cast. Large undercuts on dental specimens, which may interfere with moulding (such as underneath the teeth, between the teeth and the bone) and which were not relevant to tooth function, were filled with modelling clay. Small teeth were moulded by using a pointed probe to apply very small amounts (a few $\mathrm{mm}^{3}$ ) of silicone to both sides of the tooth row. The silicone was gently pushed up the sides of the teeth, until the entire tooth surface and some surrounding bone were covered, while ensuring that no bubbles were present. A small basin made of modelling clay was filled with silicone and the tooth row covered with silicone was inverted and placed into the basin to set for $24 \mathrm{~h}$ at room temperature. The mould was gently removed using a flattened blunt probe inserted between the mould and specimen.

To ensure a robust cast that is easy to handle, the original mould was made into a larger mould with a greater volume by embedding it in a small tray (about $25 \times 20 \times 10 \mathrm{~mm}$ ) made with Laboratory-Putty (Coltène, Whaledent, Mahwah, 

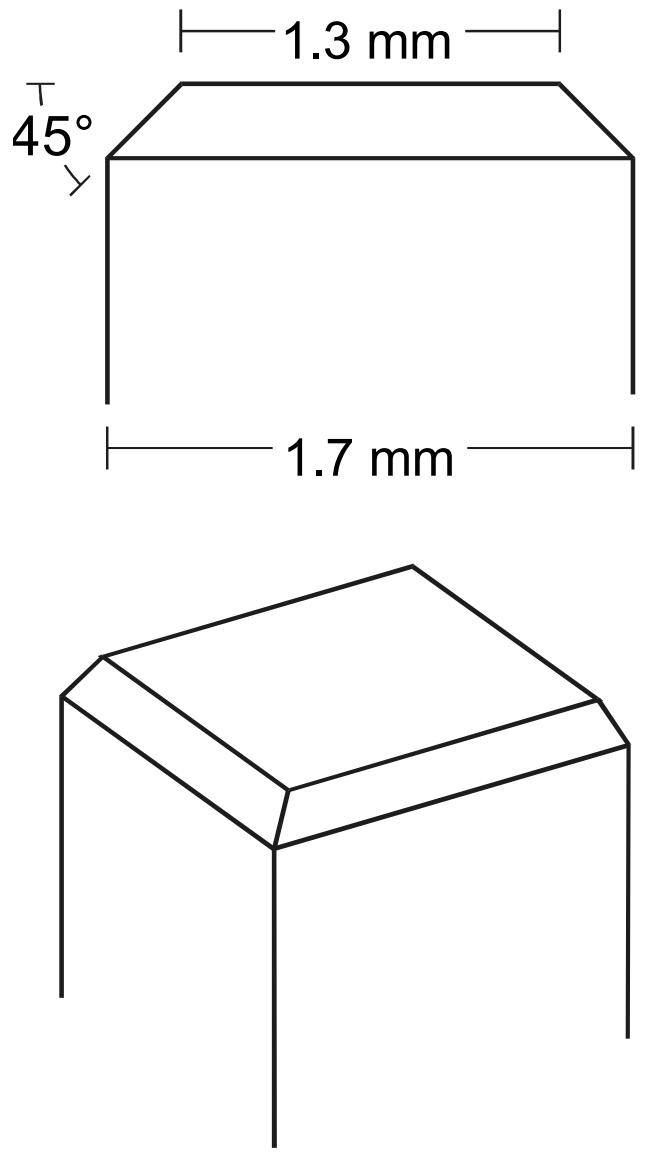

Fig. 1. Two views of the standard glass specimen for moulding, casting and imaging.

U.S.A.), with sides slanting outward to aid in later removal of the cast. The entire mould was then allowed to set.

\section{Casting}

The completed moulds were glued to microscope slides with silicone adhesive (CAF 3, Rhodorsil, Saint-Fons, France), and the slides placed into trays adapted for a hand centrifuge (Hettich Zentrifugen 1011I, Tuttlingen, Germany) which were designed to hold slides. The urethane EasyCast (British United Industries, Melbourne, Australia), which is opaque and stark white when cured, was used to make the casts. It was necessary to use a small paintbrush to eliminate most air bubbles introduced during mixing. More complex moulds (such as those of teeth) were then spun in the hand centrifuge 40 times at about 2000 rev $\min ^{-1}$. The casts were removed from the mould at the appropriate demould time $\left(25 \mathrm{~min}\right.$ at $\left.20{ }^{\circ} \mathrm{C}\right)$ and were then immediately dipped into a concentrated solution of eosin in acetone $(2 \mathrm{~g} / 100 \mathrm{~mL})$. Eosin was used as it does not fade or bleach as rapidly as fluorescein, from which it is derived. Certainly other compounds will yield equally suitable results, but eosin was chosen simply for its resin-compatibility, photostability and wide emission spectrum, which together result in a strong, stable signal. Casts were found to be sensitive to photobleaching by sunlight and so were kept in the dark when not being scanned. Laser scanning, however, did not cause significant photobleaching.

\section{Imaging}

The casts were imaged using a laser scanning confocal microscope (Leica TCS_NT, Leica, Sydney, Australia) with $\times 5(0.12 \mathrm{NA})$ and $\times 10(0.3 \mathrm{NA})$ dry objectives through rhodamine optics (excitation $568 \mathrm{~nm}$ and emission 590 long pass). The fields of view of these lenses are $2 \times 2 \mathrm{~mm}$ and $1 \times 1 \mathrm{~mm}$, respectively. The pinhole was set at 1.00 optical unit, corresponding to a measured axial resolution of $\pm 35 \mu \mathrm{m}$ and $\pm 10 \mu \mathrm{m}$, respectively. Care was taken not to oversaturate the detector when scanning. Optical slices were taken through the object in the $x, y$ plane, where each slice was a square $($ e.g. $256 \times 256)$ pixel 8 -bit image at medium scanning speed. Slices were taken at the same distance as the interval between pixels to make cubic voxels (volume pixels).

The Leica software 'PowerScan' was used to generate an 8-bit topological image from the stack of slices, where pixel intensity (grey level) represented height, by detecting the $z$ height with the greatest fluorescence for each $x, y$ point. The topological image was converted to $x, y, z$ coordinates according to the fields of view in the $x$ and $y$ directions, and the $z$ scan height. In the majority of these tests, the cubic voxels were $7.8 \mu \mathrm{m}$ long, generated in one of the following ways: for the $\times 5$ lens, either a $256 \times 256$ image was scanned at zoom 1 (field of view (FOV) of $2 \times 2 \mathrm{~mm}$ ), or a $128 \times 128$ image scanned at zoom 2 (FOV $1 \times 1 \mathrm{~mm})$; for the $\times 10$ lens, a $128 \times 128$ image scanned at zoom 1 (FOV $1 \times 1 \mathrm{~mm})$.

The two lenses were tested for the amount of surface noise in a scan. A varying amount of noise (random fluctuation in signal) is present at each pixel in a slice. As the height of the surface is judged according to the slice with the maximum signal, if noise at $z$ height $a$ is greater than signal at height $b$ (the true position of the surface), then the position of the surface is misinterpreted as being at $a$ rather than $b$. The surface noise at that point is then $|a-b|$. The surface noise level will also depend on the optical sectioning ability of the lens. With a small depth-offield (lenses with a higher numerical aperture), light from a limited range of $z$ heights will be captured, with the surface detected in, for example, only one slice. Increased depth-offield (found with lower numerical apertures) means that light from the surface is captured from a greater number of $z$ heights, and the surface is detected in many slices. As there will be random noise at each level, the slice where the signal + noise is greater than the signal + noise of every other 

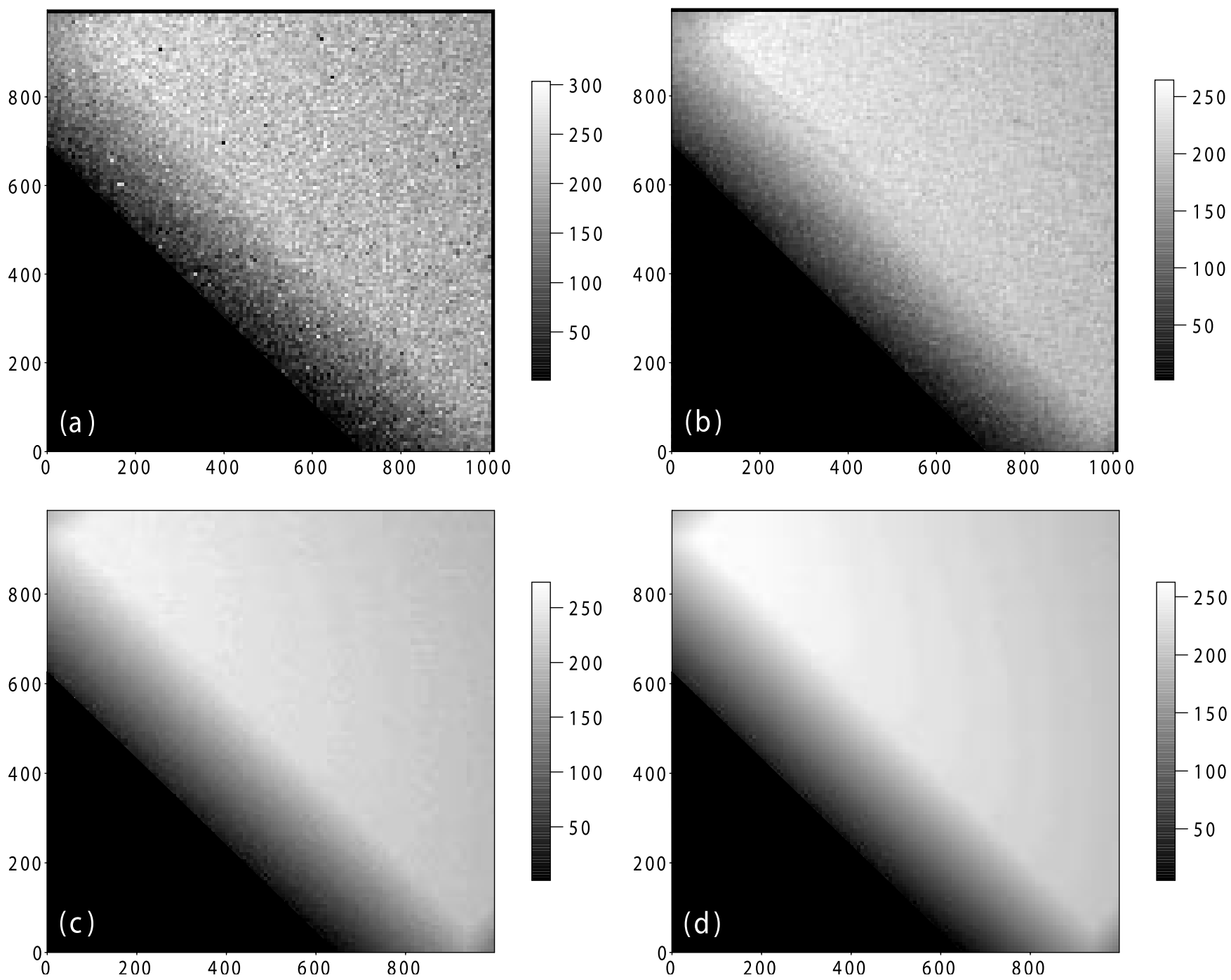

Fig. 2. Surface scans of standard glass specimen using (a) $\times 5$ lens, Accumulation 1, one scan; (b) $\times 5$, Accumulation 1 , Average of 8 topological scans; (c) $\times 10$, Accumulation 1, one scan; (d) $\times 10$, Accumulation 1, Average 8 . Dimensions in $\mu \mathrm{m}$.

Fig. 3. Relationship between number of scans and surface noise in scan (mean $\pm \mathrm{SE}$ of deviation from the plane in $\mu \mathrm{m}$ ) for two different surface noisereduction methods: Accumulation and Average (number of casts scanned $=4$ ).

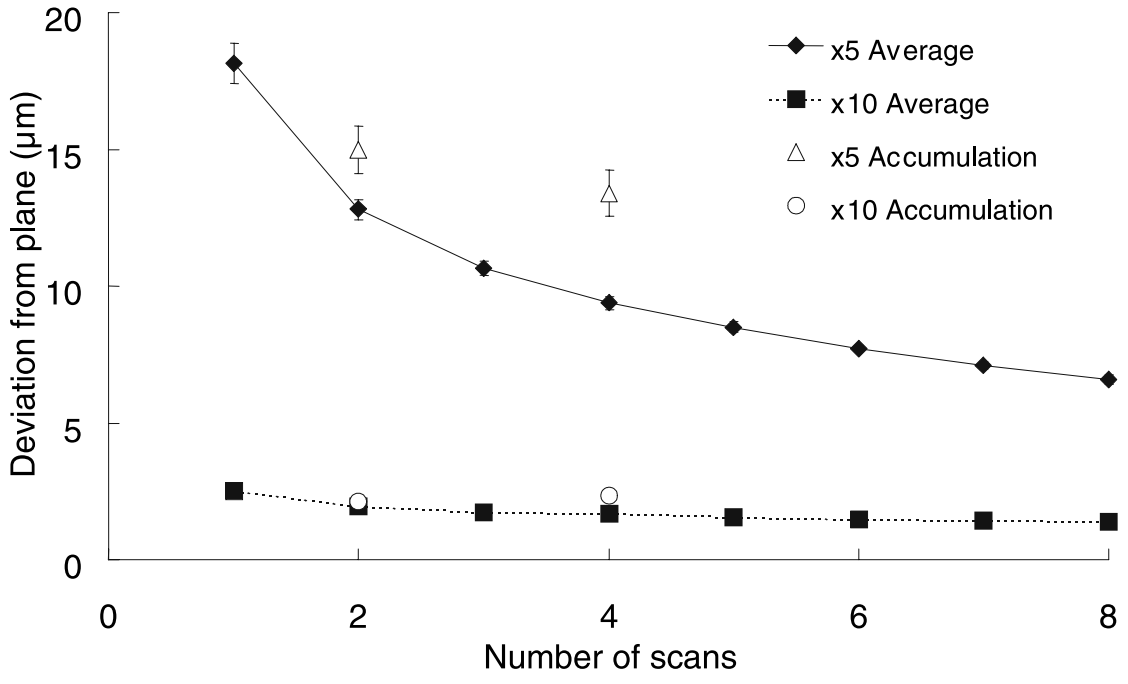



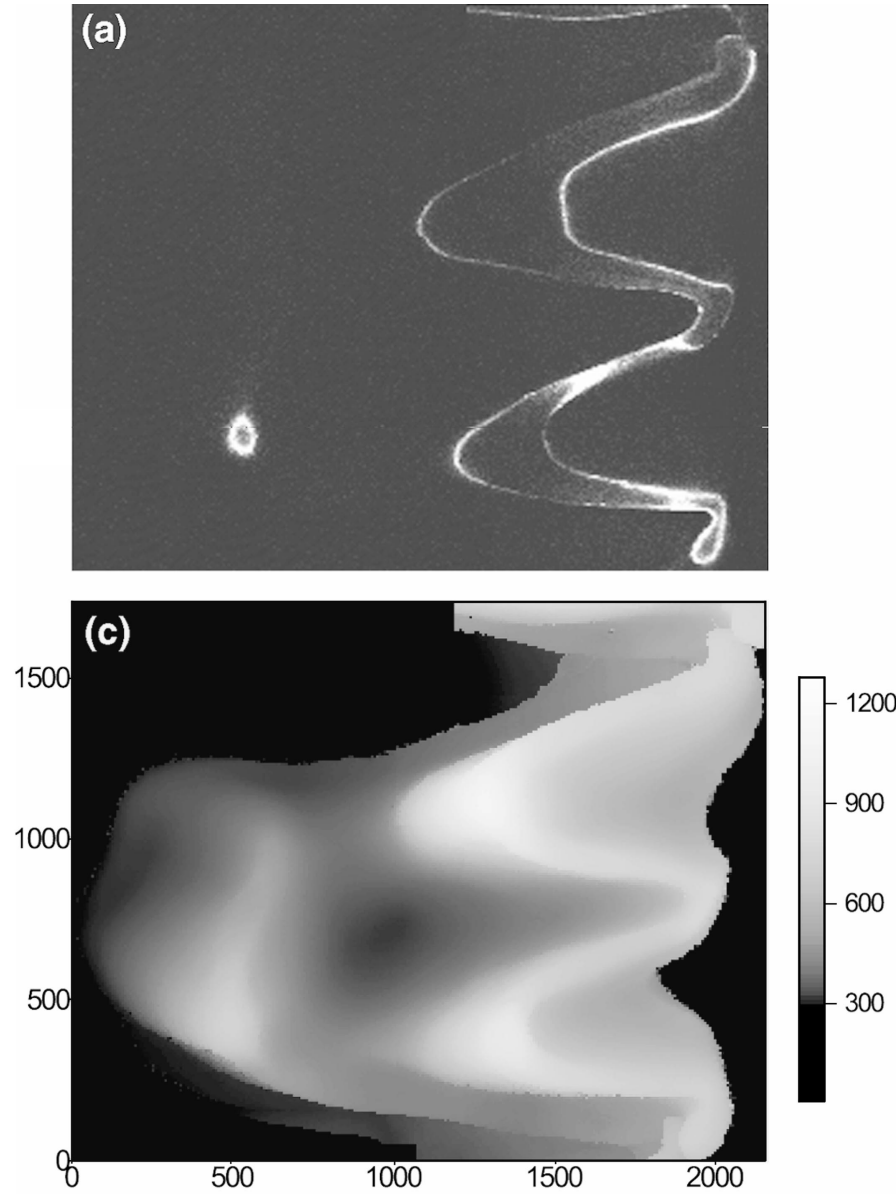
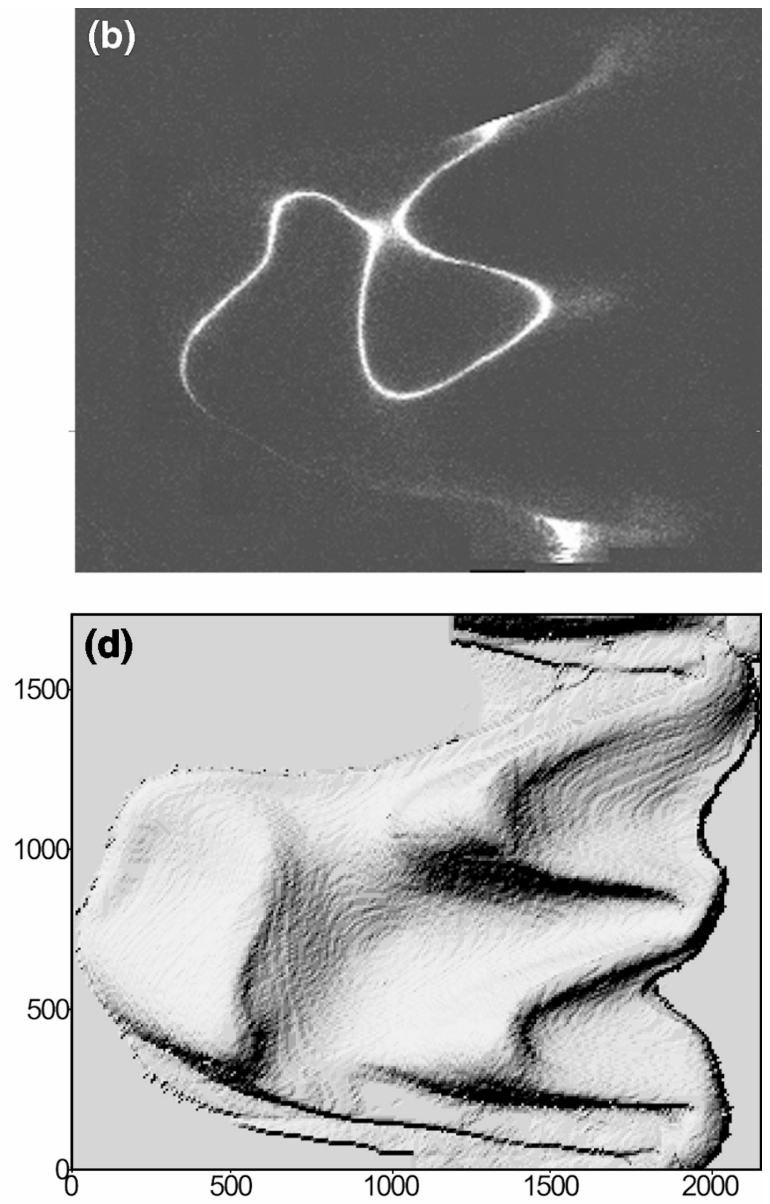

Fig. 4. Surface data representations of upper second molar of Chalinolobus gouldii: (a) and (b) optical slices through the tooth at different $\mathrm{z}$ heights, where white represents fluorescent surface; (c) topographic image, where grey level represents height; (d) shaded relief map, illustrating the tooth as a landform with light creating shadows on the surface. Dimensions in $\mu \mathrm{m}$.

slice will be the position of the surface as interpreted by the computer. There is a greater potential for misinterpretation of the surface in this case, and so the average level of surface noise will be greater.

Surface noise in a scan for any given lens may be decreased in various ways. One method is to accumulate and average several images at each $z$ height, and then create the topological image from the stack of accumulated image slices. Hence, topography created by an Accumulation 2' scan means that two images were averaged at each $z$ height. Another method is to take the average of several topological images of the same area (where 'Average 2' means two topological images have been averaged). To test which of these two gives a better image (the one with the least surface noise), final 3-D data sets of the surface were made of a cast of the standard glass specimen using each of the methods. To measure the amount of surface noise, a plane $(z=f(x, y)=a x+b y+c)$ was fitted to all the points on the top surface (usually greater than 4000 points) and this plane was subtracted from the original data. This also takes into account differences in orientation between specimens, and hence the subtracted surface is only surface noise. The mean of the deviation of each point from the plane was then measured for an area of $20 \times 20$ data points (making a standard 400 points for each scan).

Scans of the glass surface were carried out at $7.8 \mu \mathrm{m}$ cubic voxels for both $\times 5$ and $\times 10$ lenses. The same area of each cast was scanned eight times at Accumulation 1 (one scan) and twice each at Accumulations 2 and 4. Averages of two to eight scans were also generated. Surface noise was measured for each scan. Four casts, each from a different mould of the standard glass specimen, were scanned in this manner.

Urethane casts of the upper right second molar of three $C$. gouldii specimens were scanned using the $\times 10$ lens at $128 \times 128$ pixels $(7.8 \mu \mathrm{m}$ cubic voxels $)$ at Accumulation 2. The entire right upper and lower molar rows of one specimen were scanned using the $\times 5$ lens at $128 \times 128$ pixels (15.6 $\mu \mathrm{m}$ voxels) at Accumulation 4 . The tooth casts were positioned on modelling clay on a microscope slide so that as much of the occlusal surface was visible as possible, 
i.e. there were no undercuts in the scan. Because the teeth were larger than the diameter of the field of view, several topographical images were taken and then manually tiled using Scion Image (Scion Corporation, Maryland, U.S.A.).

\section{Visual representation}

Once the topological image has been generated by the Leica 'PowerScan' software (in which height is represented by the grey level), this surface may be visualized as an extended focus image or as orthogonal views through the image stack, which is useful in assessing optical sectioning ability.

Another helpful tool is "Virtual Reality Modelling Language' (VRML). VRML was originally developed for use on web pages as an alternative to 'Hypertext Markup Language' (HTML). It was designed for the simple construction of virtual objects and worlds. World builders and browsers such as 'Community Place Conductor 2.0' (Sony Corporation, Park Ridge, U.S.A.) and 'Cosmo Player 2.1' (Computer Associates International, Inc., Islandia, U.S.A.) can be used to construct and explore these worlds on the computer. It provides a simple way of representing objects in three dimensions, allowing the observer to view objects from any orientation and distance.

The command 'ElevationGrid' in VRML 97 allows the user to specify a uniform rectangular grid of varying height in the $z=0$ plane. This is ideal for representing topological images from the confocal microscope, which are $x, y$ matrices of $z$ heights. When calibrated in the $x, y$ and $z$ directions, a VRML browser such as 'Cosmo Player 2.1' can generate a correctly proportioned representation of the tooth on the screen, which can be rotated in any direction, and a simulation of walking on the tooth surface can be produced. Two ElevationGrids can be generated in the same 'virtual world', and their positions in three-dimensional space at various time points can be specified using the 'TimeSensor' and 'PositionInterpolator' commands. The browser will interpolate the position of the moving teeth between the given time points, resulting in a smooth animation of teeth moving into and out of occlusion along a specified occlusal stroke. A full list and usage of VRML 97 commands can be found at http:// www.vrml.org/Specifications.

The other main software used was the geographical information systems (GIS) program Surfer (Win 32) V 6.04 (Golden Software, Inc., Golden, U.S.A.), which is used for geological surface mapping. This software allows the final surface to be viewed as a topographic image and a shaded relief image (simulating light falling on the surface), and also be used to obtain profiles of the surface.

\section{Measurement}

Independent measurements of the glass specimen were made using a Reflex Prior 52000 3-D motorized microscope
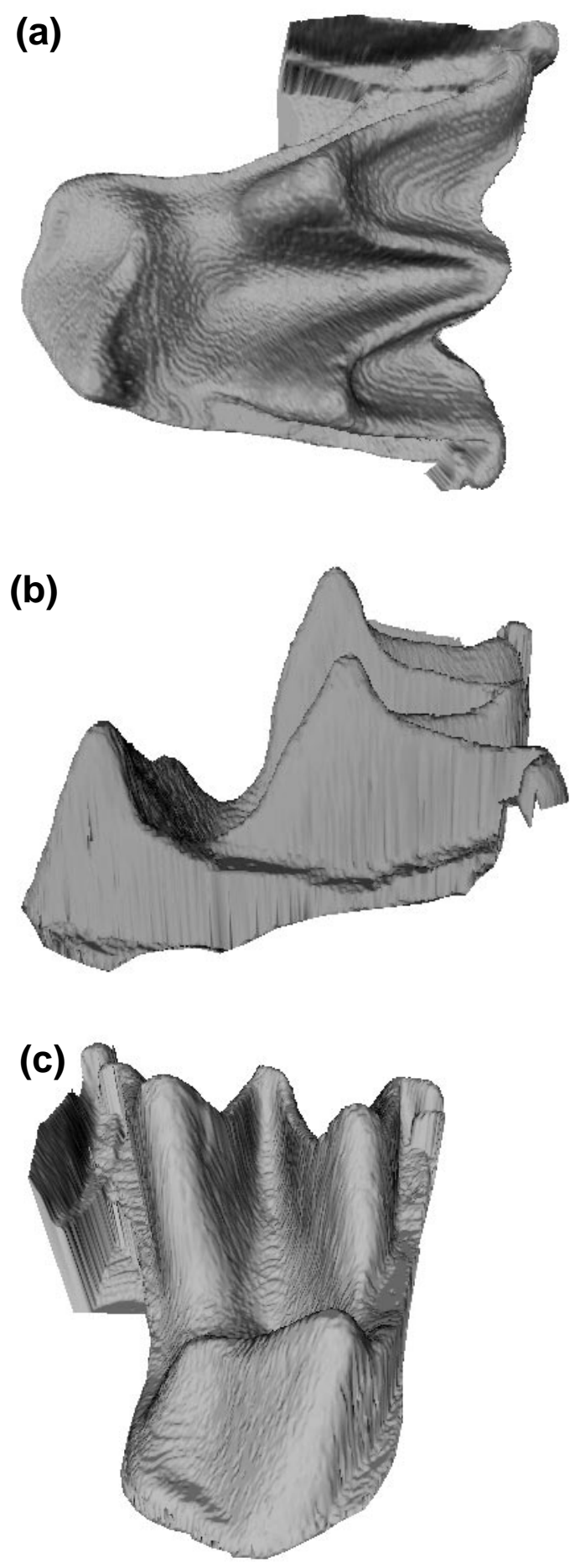

Fig. 5. Virtual reality modelling language reconstructions of upper second molar of Chalinolobus gouldii: (a) occlusal view; (b) anterior view; (c) lingual view. 
Lingual
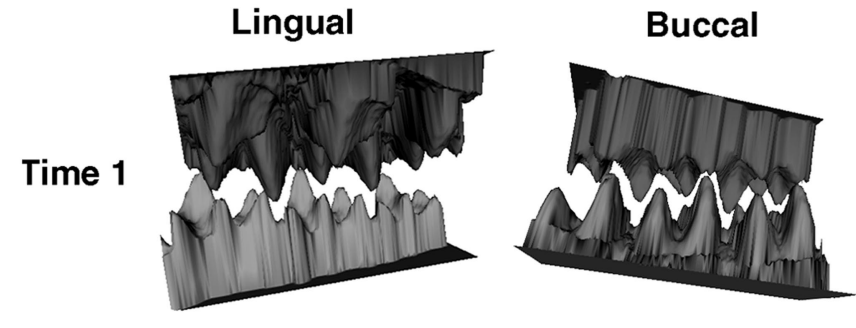

Time 2

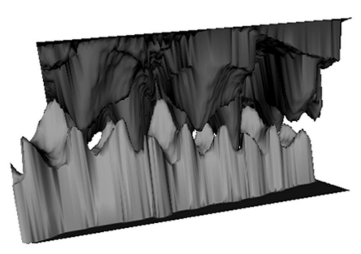

Time 3
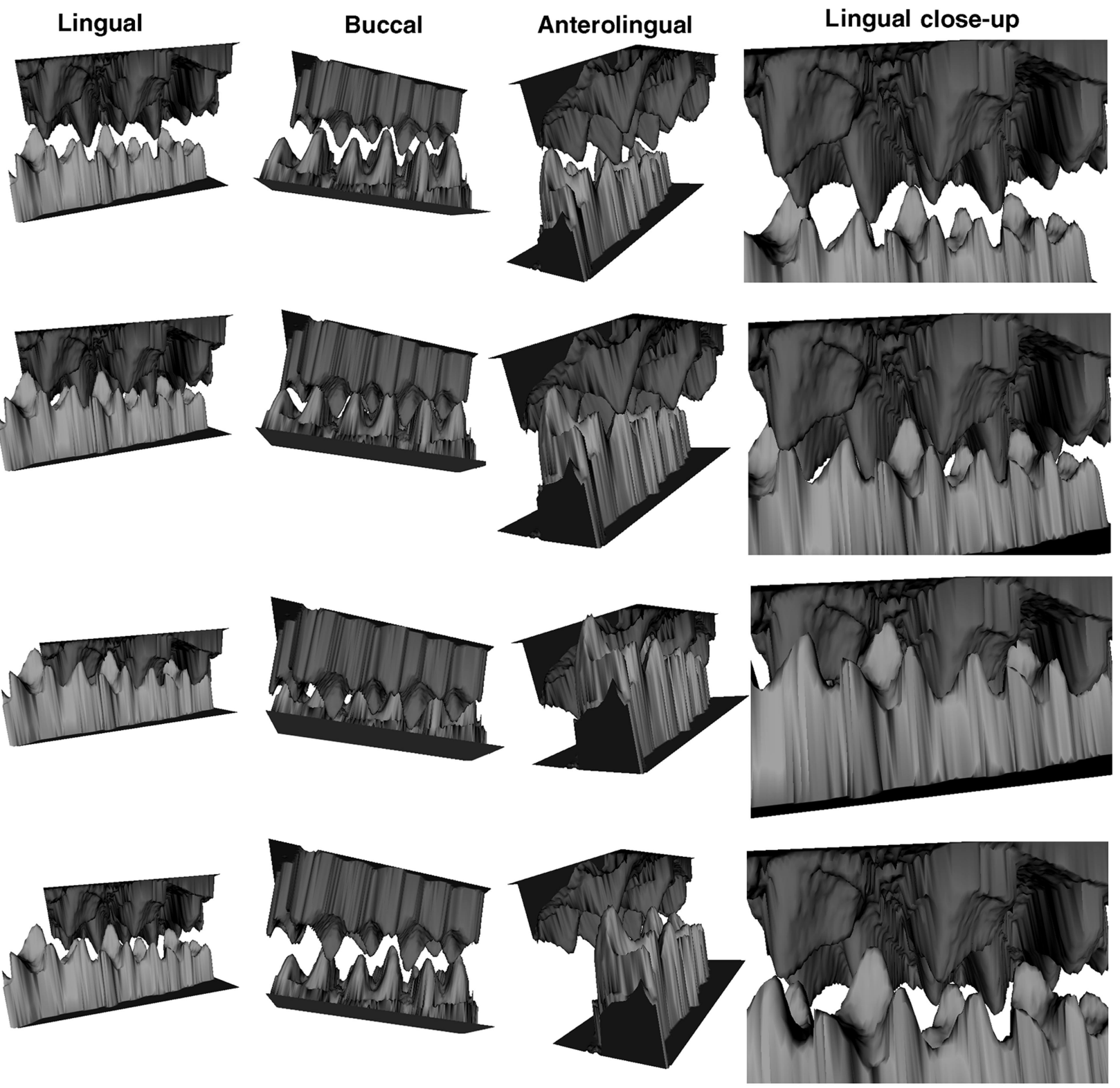

Fig. 6. Virtual reality modelling language reconstructions of occlusion of upper and lower molar rows in Chalinolobus gouldii. Four successive time points (Times 1-4) and four different views (lingual, buccal, anterolingual and a close-up from the lingual direction).

(Reflex Ltd, Somerset, U.K.), which can be used to capture manually the position of individual points in three dimensions. The length of one edge of the top face was measured using the Reflex. The angle between the top surface and the adjacent slanted face was found by measuring the specimen using a profile projector (Nikon Profile Projector V-20 A, Tokyo, Japan) at $\times 50$ magnification. Reflex measurements were also made on each of the casts.

Measurements were made from the topographic scans of the glass specimen using Surfer. Surfer allows the user to digitize a point (obtain its 3-D coordinates) on the surface when viewing the image as a shaded relief map, in which the features of the object can more readily be recognized. The three-dimensional distance between the points can then easily be calculated. A plane was fitted to each of the top and side surfaces using all points on each surface, and the angle between these planes calculated.

Various measurements were made on the scans of $C$. gouldii teeth, including the surface areas and volumes of whole teeth and of particular features (such as cusps and basins), the three-dimensional length of crests, and angles between crests. 


\section{Results}

\section{Imaging}

Topographic images of $\times 5$ and $\times 10$ scans of the glass specimen after averaging differing numbers of scans are shown in Fig. 2, where a decrease in surface noise can be seen after a number of scans is averaged. Figure 3 shows how the average deviation from a plane (i.e. surface noise) changes for differing numbers of scans using Average and Accumulation. The amount of surface noise in the scan decreased as an increasing number of scans was averaged. This is particularly obvious for the $\times 5$ lens (mean $\pm \mathrm{SE}$ $18.14 \pm 0.74 \mu \mathrm{m}$ for one scan; $6.59 \pm 0.18 \mu \mathrm{m}$ for Average $8 ; n=4)$. Surface noise did decrease to a small extent in the $\times 10$ lens $(2.53 \pm 0.17 \mu \mathrm{m}$ and $1.39 \pm 0.07 \mu \mathrm{m}$ for one and Average 8 scans, respectively), but was always substantially less than that of the $\times 5$ lens.

Scans of Accumulation 2 (14.98 $\pm 0.87 \mu \mathrm{m}$ for $\times 5$; $2.12 \pm 0.14 \mu \mathrm{m}$ for $\times 10)$ and Accumulation 4 $(13.38 \pm 0.84 \mu \mathrm{m}$ for $\times 5 ; 2.34 \pm 0.06 \mu \mathrm{m}$ for $\times 10)$ had less surface noise than a single scan (only one frame at each slice), but more than Average 2 for the same lens. This can in part be explained by the lower $z$ resolution in Accumulation. For Average, the images were first converted to $x, y, z$ data points and then averaged, and so the data were continuous in the $z$ direction; for Accumulation, the $z$ resolution was the sampling interval between stacks, and so the minimum deviation from the surface (apart from zero) was the interval between stacks.

\section{Visual representation}

Figures 4(a) and (b) show two optical slices through a tooth cast taken by fluorescence imaging. Two different visual representations of the same data are demonstrated in Figs 4(c) and (d): a topological image, where grey scale represents height; and a shaded relief map, where the surface is treated like a landscape over which light and shadow fall. All surface reconstructions presented in this paper are the raw data from the scans and have not been smoothed or interpolated. These modifications of the data may be useful for some studies and for simple visualization, but the raw data are used here to show that it is an accurate representation of the specimen. Views from different directions of a VRML reconstruction of the same tooth scan are shown in Fig. 5. Still frames from the animation of upper and lower teeth from several views at four separate times are shown in Fig. 6.

\section{Measurements}

The length of one edge of the glass specimen as measured by the Reflex was $1294.1 \pm 1.0 \mu \mathrm{m}$, and the angle between the top and side faces was $44.46 \pm 0.05^{\circ}$ (10 replicate measurements for each). This length on all stained casts was $1267.5 \pm 3.6 \mu \mathrm{m}$ (10 replicate measurements on four casts; $n=4$ ), showing a shrinkage of $2.1 \%$. Measurements on unstained casts showed the casts were the same length, meaning that dipping a cast in acetone did not affect its dimensions.

Measurements of the digital surface showed a side length of $1247.7 \pm 2.3 \mu \mathrm{m}$, a difference of $3.6 \%$ compared to the original specimen. The angle between the top and side surfaces was $43.51 \pm 0.18^{\circ}$, a difference of $0.95^{\circ}$.

A summary of some measurements taken from the bat tooth scans is shown in Table 1. Specimens generally increased in size (length and surface area measurements) in the following order: C3748, C3746, C3747.

\section{Discussion}

Confocal microscopy is useful for studying tooth surfaces, particularly molar and premolar tooth surfaces, as it can only be used for those macro surfaces that do not have undercuts. Full 3-D rendered models can be created of much smaller objects (e.g. whole cells or organelles), but cannot be done for larger objects using this method. There is a significant loss of signal intensity with increasing penetration into the specimen due to both absorption and scattering of the excitation beam on its way to the optical focus plane, and this applies also to the emitted light between the focus plane and detector (Cheng \& Kriete, 1995). Additionally, any optical heterogeneity of the specimen will further degrade the signal, thus ensuring that undercut surfaces outside this level cannot be detected and reconstructed with any accuracy, regardless of the reconstruction software used. However, the entire surface of the molars of most animals, including the smallest, tribosphenic molars, can be imaged from at least one perspective without significant undercuts.

However, this does present a problem for more vertically extended teeth, such as canines, which often curve back on themselves. A full three-dimensional model of such teeth

Table 1. Measurements from scans of upper second molar of three specimens of Chalinolobus gouldii taken from the digital model using 'Surfer'.

\begin{tabular}{llll}
\hline & $\begin{array}{l}\text { Tooth } \\
\text { length }(\mu \mathrm{m})\end{array}$ & $\begin{array}{l}\text { 3-D length } \\
\text { of crests }(\mu \mathrm{m})\end{array}$ & $\begin{array}{l}\text { Tooth surface } \\
\text { area }\left(\mathrm{mm}^{2}\right)\end{array}$ \\
\hline C3746 & 1368.4 & 9305.5 & 6.16 \\
C3747 & 1382.3 & 9433.8 & 6.32 \\
C3748 & 1335.8 & 8810.9 & 5.98 \\
\hline
\end{tabular}


can be constructed using the surface data from several views, but fitting together multiple views, and manipulation of the surface once it is constructed (which is usually in the form of polygons to represent the surface) is more complex. Another benefit is the higher resolution that can be obtained by confocal microscopy compared to many digital scanning devices currently available: e.g. the Surveyor 500 (Laser Design, Inc., Minneapolis, U.S.A.) used by Ungar \& Williamson (2000) has a maximum resolution of $25.4 \mu \mathrm{m}$ in the three orthogonal directions. For a good representation of a small mammalian tooth, this is probably too large. Laser scanning confocal microscopy can reach resolutions of fractions of micrometres, but for an object the size of a tooth the resolution would be unnecessarily high, increasing data gathering and computational time.

The imaging method has been shown to be consistent over the various stages of data gathering - scans of the same cast and scans of casts from different moulds are all very similar. This gives us confidence that taking one scan from one cast of a mould should be representative of the original object, and so multiple moulds, casts and scans are not required, minimizing the time needed to gather data.

The published statistics on shrinkage for the materials used show an expected shrinkage of $0.2-0.4 \%$ for RTV 3110 and $1.0 \%$ for EasyCast. This means that for an object moulded and cast from both materials, shrinkage should be at most $2 \%$. This is approximately the result that we obtained.

A number of different casting and moulding materials were tested for their use in fluorescence imaging of macro objects. Epoxy plastics were found to be inferior to the urethane in several respects. Epoxies are usually translucent and when the dye was incorporated into the cast a fluorescent signal from inside the cast was often detected and the position of the surface misinterpreted, especially at higher dye concentrations. At lower dye concentrations this was generally not a problem, but the surface noise was often higher than that for urethane, probably due to the signal being detected from a short distance inside the cast. The urethane used in this study is opaque, and hence the maximum fluorescence is detected from the surface only.

Epoxies can be highly corrosive to silicone moulding materials: only three or four casts may be made before the mould is significantly damaged, and even the first cast can show signs of deterioration (e.g. Araldite M (Ciba-Geigy, Melbourne, Australia) caused deterioration of RTV 3110 and CAF 3 moulds (A. R. Evans, personal observation); moulding and casting artefacts have been found by Gordon, 1984 and Teaford \& Oyen, 1989). Many casts (> 20) can be made from silicone moulds when urethane is used. The main disadvantage of urethane compared to epoxy is the lower dimensional stability of urethane. Many epoxies have published shrinkages of $0.01 \%$ compared to $1 \%$ of urethane. It was considered, however, that the superior imaging of the urethane casts would be of a greater advantage than a low shrinkage on a cast that may have defects due to the nature of the casting agent.

It is not known how the scanning accuracy (surface noise level) using other scanning and casting techniques compares with that reported here. Jernvall \& Selänne (1999) report that three to six scans were averaged to achieve a final topographic image. Surface noise levels have not been published for 3-D scanning devices such as the Surveyor 500.

The difference in the amount of surface noise between the two lenses is extremely obvious, as would be predicted from their numerical aperture. The benefits of averaging multiple scans are also apparent, but the results here show that additional scanning does not always reduce noise. For example, very little surface noise reduction is gained from averaging more than two scans in the case of the $\times 10$ lens. The scans of tooth casts with this lens were carried out using Accumulation 2 rather than Average 2 due to the necessity of tiling multiple images, as averaging tiled topological images would be tedious. There is a significant decrease in surface noise when using both averaging and accumulation with the $\times 5$ lens. Accumulation 4 was used for scans of the entire tooth row made using this lens, which showed a large reduction in surface noise compared to Accumulation 1 . The $\times 5$ lens was used for tooth row scans due to the much larger field of view diameter to reduce the number of scans necessary to cover the area.

As the main application of this work is to analyse large numbers of teeth, an important consideration in deciding which lens to use is the scanning time. There is a trade-off between resolution (determined by the number of pixels), reduction of surface noise and time taken to scan. Measured scan times (Table 2) demonstrate that the time to scan one slice is simply related to the resolution and the number of scans accumulated. Scanning a $C$. gouldii $\mathrm{M}^{2}$ of $2.2 \times 1.7 \mathrm{~mm}$ using $\times 10$ lens required five separate scans of 170 sections each to cover the area at voxel $7.8 \mu \mathrm{m}$ and took a total of $19 \mathrm{~min} 10 \mathrm{~s}$. The scans of the entire molar tooth row at voxel $15.6 \mu \mathrm{m}$ with $\times 5$ lens required three scans of about 115 slices (total of $15: 30$ ).

Scan times will also help to decide whether any improvement in the optical sectioning of the $\times 10$ lens is offset by its smaller field of view. For the set-up used here, the same area and resolution is covered by $\times 5$ lens at 1.0 s slice $^{-1}$ compared to $4 \times 0.7 \mathrm{~s}$ slice ${ }^{-1}=2.8 \mathrm{~s} \mathrm{slice}^{-1}$ for the $\times 10$ lens, not including the time to reposition the object between scans. However, if the more important consideration is accuracy (reducing surface noise), then the $\times 10$ lens would be better, given that an equivalent scan time gives a lower surface noise (Accumulation $4, \times 5$, gives surface noise of $13.38 \mu \mathrm{m}$ and takes $4.1 \mathrm{~s} \mathrm{slice}^{-1}$; Accumulation $1, \times 10$, gives surface noise of $2.53 \mu \mathrm{m}$ and takes $2.8 \mathrm{~s} \mathrm{slice}^{-1}$ for the same area). 
Table 2. Relationship between resolution, number of scans accumulated at each $\mathrm{z}$ height and the time taken to scan. Results are the same for both $\times 5$ and $\times 10$ lenses.

\begin{tabular}{lllll}
\hline $\begin{array}{l}\text { Resolution } \\
\text { (pixels } \times \text { pixels) }\end{array}$ & $\begin{array}{l}\text { No. frames } \\
\text { accumulated }\end{array}$ & $\begin{array}{l}\text { Time to scan } \\
100 \text { slices }(\mathrm{s})\end{array}$ & $\begin{array}{l}\text { Time per } \\
\text { slice }(\mathrm{s})\end{array}$ & $\begin{array}{l}\text { Time per } \\
\text { scan (s) }\end{array}$ \\
\hline $128 \times 128$ & 1 & 68.75 & 0.69 & 0.69 \\
$128 \times 128$ & 2 & 136.4 & 1.36 & 0.68 \\
$128 \times 128$ & 4 & 271.6 & 2.72 & 0.68 \\
$256 \times 256$ & 1 & 103.3 & 1.03 & 1.03 \\
$256 \times 256$ & 2 & 204.1 & 2.04 & 1.02 \\
$256 \times 256$ & 4 & 406.7 & 4.07 & 1.02 \\
\hline
\end{tabular}

Scans of whole teeth can then be made at the same resolution with lower surface noise using $\times 10$ without a major increase in scanning time. These comparisons can be made for any microscope set-up once data on scan times and surface noise are obtained.

Using these methods, many measurements of the digitized teeth are currently possible (Table 1), including length, area and volume measurements. From these surface data, it is now possible to make measurements of surface sharpness (Evans \& Sanson, 2000), which is important to tooth function (Freeman, 1992; Popowics \& Fortelius, 1997; Evans \& Sanson, 1998). Comparisons of these and other such measurements will be very important when dissecting out the differences in shape and therefore function in teeth of all forms.

Understanding the intricate arrangement of cusps and crests on a single tribosphenic tooth is very difficult, especially when only line drawings or photographs are available. The small size of the teeth also means that they must be viewed through a microscope. However, once digitized, the visualization can be carried out on the computer. A tooth represented in a VRML file (such as that shown in Fig. 5) can allow the viewer to fully grasp the three-dimensional shape of the tooth.

Reconstructing the occlusion of upper and lower tribosphenic teeth is also difficult and tedious for functional morphologists and students alike. In previous work on tooth function, the spatial relationships between tooth features could only be understood by embedding casts in resin at various stages of occlusion and then sectioning them (e.g. Crompton \& Hiiemae, 1970). The same representations can now be done on the computer by digital manipulation of the surface data of teeth, involving much less guesswork in orienting teeth and labour in making and sectioning casts.

From the reconstruction of tooth movement during the occlusal stroke, as seen in Fig. 6, the major events of tooth occlusion can be seen: the protoconid (the largest cusp on the lower molars) fits in the embrasure between the uppers; shearing occurs along the two crests leading down from the protoconid against crests on the upper teeth; and the lingual cusp on the upper tooth (protocone) fits into the talonid basin in the lower. Another consideration that is important but difficult to visualize when viewing two isolated teeth is that the lower tooth must move lingually during the occlusal stroke in order for these events to occur.

The advantages of digital reconstruction are very apparent, especially when considering small objects such as mammalian teeth. Conventional methods of visualizing how the upper and lower molars fit together for teeth less than $2 \mathrm{~mm}$ in length are extremely difficult and can be misleading. These advantages were seen to outweigh the slightly greater inaccuracy in measurements taken from the confocal compared to the Reflex microscope (a difference of less than $4 \%$ ) - the benefits of the techniques greatly outweigh the costs (as similarly found in other studies comparing confocal and conventional microscopy accuracy, e.g. Delorme et al., 1998). Future digitizing techniques for small objects will easily overcome these drawbacks, and the methods of surface data manipulation described here will become an even greater part of the study of morphology than at present. All problems relating to the digitization of macro objects are not solved by confocal microscopy, as it is incapable of such reconstructions as a whole skull. However, the present study shows it to be very effective for small objects (millimetre size range), such as teeth.

\section{Acknowledgements}

Thanks to Lesley Kool, Department of Earth Sciences, Monash University, for advice on moulding and casting, and Lina Frigo, Mammalogy, Museum Victoria, for loan of specimens. Alistair Evans is supported by a Monash Graduate Scholarship.

\section{Supplementary material}

The following material is available from http://www.blackwell-science.com/products/journals/suppmat/JMS/

JMS939/JMS939sm.htm

Fig. S1. Animated view of optical slices stack through upper second molar of Chalinolobus gouldii.

Fig. S2. Virtual Reality Modelling Language reconstruction of upper second molar of Chalinolobus gouldii.

Fig. S3. Video of Virtual Reality Modelling Language reconstruction of occlusion of upper and lower molar rows of Chalinolobus gouldii.

Fig. S4. Animated Virtual Reality Modelling Language reconstruction of occlusion of upper and lower molar rows of Chalinolobus gouldii.

Figure S1 is an animated gif of successive slices through the tooth. Figure S2 is the VRML reconstruction where the tooth can be viewed from any direction. Figure S3 shows a colour video of tooth occlusion from one view; the animated 
VRML file that can be viewed from all directions is shown in Figure S4.

\section{References}

Boyde, A. \& Fortelius, M. (1991) New confocal LM method for studying local relative microrelief with special reference to wear studies. Scanning, 13, 429-430.

Boyde, A. \& Jones, S.J. (1995) Mapping and measuring surfaces using reflection confocal microscopy. Handbook of Biological Confocal Microscopy (ed. by J. B. Pawley), pp. 255-266. Plenum Press, New York.

Cheng, P.C. \& Kriete, A. (1995) Image contrast in confocal light microscopy. Handbook of Biological Confocal Microscopy (ed. by J. B. Pawley), pp. 281-310. Plenum Press, New York.

Crompton, A.W. \& Hiiemae, K. (1970) Molar occlusion and mandibular movements during occlusion in the American opossum, Didelphis marsupialis L. Zool. J. Lin. Soc. 49, 21-47.

Delorme, R., Benchaib, M., Bryon, P.A. \& Souchier, C. (1998) Measurement accuracy in confocal microscopy. J. Microsc. 192, $151-162$.

Evans, A.R., Harper, I.S. \& Sanson, G.D. (1999) Methods of digital measurement of tooth surface and occlusion. Abstracts 11th Int. Conf Confocal Microscopy, European Mol Biol. Laboratory (EMBL), Heidelberg, Germany, 11-15 April 1999, p. 23.

Evans, A.R. \& Sanson, G.D. (1998) The effect of tooth shape on the breakdown of insects. J. Zool. (Lond.), 246, 391-400.

Evans, A.R. \& Sanson, G.D. (2000) Applications of confocal microscopy to functional morphology: the effect of diet and wear on tooth sharpness and function in two microchiropterans Chalinolobus gouldii and C. morio. Proceedings of the 9th Australasian Bat Conference, Tocal, Australia, 25-28 April 2000. Bat Res. News, 41, 44.
Freeman, P.W. (1992) Canine teeth of bats (Microchiroptera): size, shape and role in crack propagation. Biol. J. Lin. Soc. 45, 97115.

Gordon, K.D. (1984) Pitting and bubbling artefacts in surface replicas made with silicone elastomers. J. Microsc. 134, 183188.

Hunter, J.P. \& Jernvall, J. (1998) Decomposing early morphological diversity in ungulates: analysis of early Paleocene arctocyonid teeth. J. Vertebrate Paleontol. 18, 52A.

Jernvall, J. \& Selänne, L. (1999) Laser confocal microscopy and geographic information systems in the study of dental morphology. Palaeontologia Electronica, 2. http://palaeo-electronica.org.

Popowics, T.E. \& Fortelius, M. (1997) On the cutting edge: tooth blade sharpness in herbivorous and faunivorous mammals. Ann. Zool. Fennici, 34, 73-88.

Reed, D.N.O. (1997) Contour mapping as a new method for interpreting diet from tooth morphology. Am. J. Phys. Anthropol. Supplement, 24, 194.

Teaford, M.F. \& Oyen, O.J. (1989) Live primates and dental replication: new problems and new techniques. Am. J. Phys. Anthropol. 80, 73-81.

Ungar, P. \& Williamson, M. (2000) Exploring the effects of toothwear on functional morphology: a preliminary study using dental topographic analysis. Palaeontologia Electronica, 3. http:// palaeo-electronica.org/2000_1/gorilla/issue1_100.htm.

Valeri, C.J., Cole, T.M. III, Lele, S. \& Richtsmeier, J.T. (1998) Capturing data from three-dimensional surfaces using fuzzy landmarks. Am. J. Phys. Anthropol. 107, 113-124.

Zuccotti, L.F., Williamson, M.D., Limp, W.F. \& Ungar, P.S. (1998) Modeling primate occlusal topography using geographic information systems technology. Am. J. Phys. Anthropol. 107, 137142. 